\title{
Microscopy-based Multi-technique, Multi-scale Characterization of Polymer Electrolyte Membrane Devices
}

Sarah Zaccarine ${ }^{1}$, Scott Mauger ${ }^{2}$, William McNeary ${ }^{2}$, Alan Weimer ${ }^{3}$, Shaun M. Alia ${ }^{2}$, Meital Shviro ${ }^{4}$, Marcelo Carmo ${ }^{4}$, Bryan S. Pivovar ${ }^{2}$ and Svitlana Pylypenko ${ }^{1}$

${ }^{1}$ Colorado School of Mines, Golden, Colorado, United States, ${ }^{2}$ National Renewable Energy Laboratory, Golden, Colorado, United States, ${ }^{3}$ University of Colorado Boulder, Boulder, Colorado, United States, ${ }^{4}$ Forschungszentrum Juelich GmbH, Julich, Nordrhein-Westfalen, Germany

Electrochemical energy devices including fuel cells and electrolyzers contain the potential to meet escalating global energy demands as a cleaner alternative to fossil fuels or as a component in a more environmentally friendly combination. Typical polymer electrolyte membrane (PEM) fuel cells (PEMFCs) and water electrolyzers (PEMWEs) are comprised of several layers of different materials that form multicomponent interfaces to promote ion transport, gas transport, and liquid flow. ${ }^{1}$ In the center of the device is the proton-conducting membrane, on either side of which are the anode and cathode catalyst layers (CLs), which are composed of three primary materials: the transition metal catalyst; carbon to provide electronic conductivity; and ionomer to bind the CL together and provide proton conductivity. Together the membrane and CLs form the membrane electrode assembly (MEA), which is the heart of PEM devices. While capable of renewable energy production, these devices face slow kinetics in their oxygen-pertaining reactions, which restricts widespread implementation. State-of-the-art catalysts used to address these concerns are noble metal-based, leading to high costs. Many catalytic approaches have been explored for both devices. In PEMFCs, the field of catalyst development has included Pt-based alloys, shaped nanoparticles, and core-shell structures. Extended surface PtNi nanowires (NWs) offer the compromise of improved activity and durability compared to other catalytic approaches and can be synthesized by scalable routes. ${ }^{2,3}$ In PEMWEs, Ir and $\mathrm{IrO}_{2}$ are standard catalysts, with other metallic and bimetallic alternatives also being researched. Each novel catalyst interacts differently with other components of the electrode layer, necessitating optimization of all device components and interactions within the fuel cell or electrolyzer.

The multi-component interfaces and interactions across various layers of these devices provide a significant characterization challenge and require a multi-technique, multi-scale suite of characterization techniques to probe various properties and interactions in order to gain a comprehensive understanding of these systems and improve efficiency and durability during device operation. Microscopy lies at the center of device characterization due to its visualization capabilities that can be used to image the catalyst at all relevant stages as it transitions from catalyst in a powder form, to ink, to CL, to fresh MEA, to tested MEA. Scanning electron microscopy (SEM), transmission electron microscopy (TEM), and scanning TEM (STEM) can be used to image both catalyst powder as well as MEAs, particularly cross-sectioned electrodes produced using ultramicrotomy, which can lead to remarkable insights into catalyst, carbon, and ionomer distribution and structure across the catalyst layer, as well as migration or agglomeration resulting from MEA operation. STEM can further be operated using energy dispersive x-ray spectroscopy (EDS) hypermapping to map elemental distribution. Combined, these microscopy techniques can provide top-down and cross-sectioned information about surface and bulk morphology from $\mu \mathrm{m}$ and $\mathrm{nm}$ (SEM, TEM) down to atomic scale information (high-resolution TEM). X-ray based microscopy and electron and $\mathrm{X}$-ray tomography take imaging capabilities a step further by enabling 3D visualization of full MEAs 
to reconstruct catalyst, carbon, and ionomer distribution across overall catalyst layers. ${ }^{5}$ Complementary chemical and structural information can be obtained through the use of $\mathrm{x}$-ray spectroscopies and diffraction/scattering experiments, including surface-sensitive x-ray photoelectron spectroscopy (XPS), quantitative bulk x-ray absorption spectroscopy (XAS), and bulk x-ray diffraction (XRD). Results can then be correlated to performance data collected using electrochemical characterization methods, including rotating disk electrode (RDE) testing of catalyst powders and MEA testing. The heterogeneity of the materials, the challenges in collecting and interpreting data, and the overall complexity in identifying the relationship between catalyst properties, ink and electrode fabrication processing variables, and the resulting electrode properties necessitate a systematic approach to understand each piece of the puzzle as complexity is increased.

This talk will cover recent developments of both fuel cell and electrolyzer catalysts and catalyst layers, and the vital role of each technique in obtaining a comprehensive picture of how to improve constituent interactions within the device. First, the importance of microscopy characterization will be demonstrated for Pt-based extended surface nanowires investigated as a promising class of PEMFC catalysts. These catalysts were synthesized using atomic layer deposition (ALD) as a controllable, scalable route. Differences between PtNi and PtNiCo samples will be discussed and compared to previously reported catalysts. Then, examples of microscopy investigations for fundamental understanding of degradation mechanism will be shown, highlighting work with Ir-based catalysts for PEMWEs. Results will be presented for all relevant stages as the catalyst transitions from a powder to incorporation into a device. Conclusions will demonstrate how combining techniques and comparing results to performance data is necessary to optimize interfaces and interactions with the introduction of a novel catalyst. The studies presented will offer further insight into how a diverse characterization approach can be used to develop composition-structure-performance relationships during each stage of MEA development in order to optimize next generation materials for various electrochemical devices.
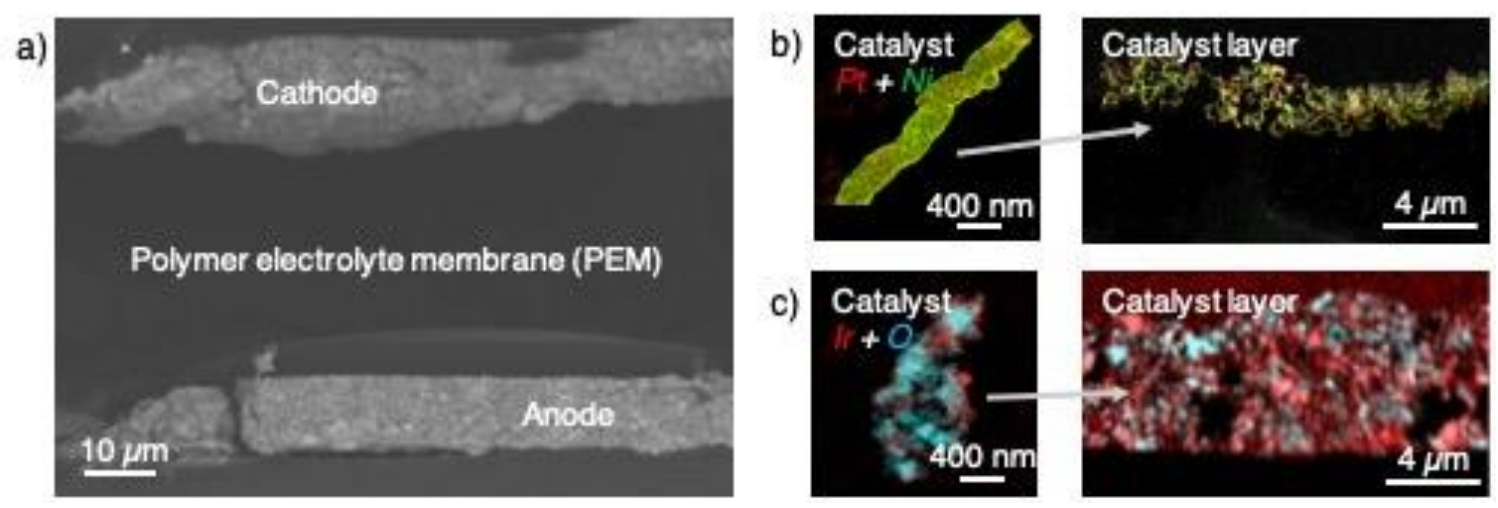

Figure 1. (a) Typical SEM cross section of an MEA for PEM electrolyzer. (b) Extended surface PtNi nanowire catalyst and catalyst layer for PEM fuel cell. (c) Ir nanoparticle catalyst and catalyst layer for PEM electrolyzer.

\section{References}

(1) Kocha, S.; Pivovar, B.; Gennett, T. Fuel Cells. In Fundamentals of Materials for Energy and Environmental Sustainability; Ginley, D. S., Cahen, D., Eds.; Cambridge University Press: Cambridge, 2011; pp 637-655. 
(2) Alia, S. M.; Ngo, C.; Shulda, S.; Ha, M.-A.; Dameron, A. A.; Weker, J. N.; Neyerlin, K. C.; Kocha, S. S.; Pylypenko, S.; Pivovar, B. S. Exceptional Oxygen Reduction Reaction Activity and Durability of Platinum-Nickel Nanowires through Synthesis and Post-Treatment Optimization. 2017.

(3) McNeary, W. W.; Ngo, C.; Linico, A. E.; Zack, J. W.; Roman, A. M.; Hurst, K. M.; Alia, S. M.; Medlin, J. W.; Pylypenko, S.; Pivovar, B. S.; Weimer, A. W. Extended Thin-Film Electrocatalyst Structures via Pt Atomic Layer Deposition. ACS Appl. Nano Mater. 2018, 1 (11), 6150-6158.

(4) Ngo, C.; Dzara, M. J.; Shulda, S.; Pylypenko, S. Spectroscopy and Microscopy for Characterization of Fuel Cell Catalysts. In Electrocatalysts for Low Temeprature Fuel Cells: Fundamentals and Recent Trends; Maiyalagan, T., Saji, V. S., Eds.; Wiley-VCH Verlag, 2017; pp 443-466.

(5) Holzer, L.; Indutnyi, F.; Gasser, P. H.; Münch, B.; Wegmann, M. Three-Dimensional Analysis of Porous BaTiO3 Ceramics Using FIB Nanotomography. J. Microsc. 2004, 216 (1), 84-95. 